\title{
Fuzzy production planning models for an unreliable production system with fuzzy production rate and stochastic/fuzzy demand rate
}

\author{
K. A. Halim ${ }^{\text {a }}$ B. C. Giri ${ }^{\mathrm{a}}$ and K. S. Chaudhuri ${ }^{\mathrm{a}}$
}

${ }^{a}$ Department of Mathematics,Jadavpur University, Kolkata 700 032, India

\section{A R T I C L E I N F O}

Article history:

Received 1 May 2010

Received in revised form

8 July 2010

Accepted 9 July 2010

Available online 9 July 2010

Keywords:

Inventory

Production planning

Imperfect production

Fuzzy number

Graded mean integration

representation method

\begin{abstract}
A B S T R A C T
In this article, we consider a single-unit unreliable production system which produces a single item. During a production run, the production process may shift from the in-control state to the out-of-control state at any random time when it produces some defective items. The defective item production rate is assumed to be imprecise and is characterized by a trapezoidal fuzzy number. The production rate is proportional to the demand rate where the proportionality constant is taken to be a fuzzy number. Two production planning models are developed on the basis of fuzzy and stochastic demand patterns. The expected cost per unit time in the fuzzy sense is derived in each model and defuzzified by using the graded mean integration representation method. Numerical examples are provided to illustrate the optimal results of the proposed fuzzy models.
\end{abstract}

(c) 2010 Growing Science Ltd. All rights reserved.

\section{Introduction}

Inventory represents an important asset to any business organization. After the pioneering work by Harris (1915) who developed the classical economic order quantity (EOQ) model with known constant demand, a great deal of researches on inventory modeling have been conducted to capture many interesting and realistic situations. However, in real world inventory systems, there exist parameters and variables which are uncertain or almost uncertain. When these uncertainties are significant, they are usually treated by probability theory. Of course, to address such an uncertainty, we need to prescribe an appropriate probability distribution. In some cases, uncertainties can be defined as fuzziness or vagueness, which are characterized by fuzzy numbers of the fuzzy set theory. Zadeh (1965) introduced fuzzy set theory to deal with quality-related problems with imprecise demand. Bellman and Zadeh (1970) distinguished the difference between randomness and fuzziness by showing that the former deals with uncertainty regarding membership or non-membership of an

* Corresponding author. Tel./fax: +91 3324146717.

E-mail addresses: bibhas_pnu@yahoo.com (B. C. Giri),

(c) 2010 Growing Science Ltd. All rights reserved. doi: 10.5267/j.ijiec.2010.04.001 
element in a set while later is concerned with the degree of uncertainty by which an element belongs to a set. In an inventory control model, Petrovic and Sweeney (1994) fuzzified the demand, lead time and inventory level into triangular fuzzy numbers. They used the fuzzy proposition method to obtain the optimal order quantity. Ishii and Konno (1998) introduced fuzziness in shortage cost by an Lshape fuzzy number when demand is stochastic. Gen et al. (1997) expressed the input data by fuzzy numbers, where they used interval mean value concept to solve an inventory problem. Yao and Chiang (2003) considered an inventory model with total demand and storing cost as triangular fuzzy numbers. They performed the defuzzification by centroid and signed distance methods. Mondal and Maiti (2002) applied genetic algorithms (GAs) to solve a multi-item fuzzy EOQ model. Maiti and Maiti (2006) dealt with a fuzzy inventory model with two warehouses under possibility constraints. Mahapatra and Maiti (2006) formulated a multi-item, multi-objective inventory model for deteriorating items with stock- and time-dependent demand rate over a finite time horizon in fuzzy stochastic environment. Halim et al. (2008) developed a fuzzy inventory model for perishable items with stochastic demand, partial backlogging and fuzzy deterioration rate. The model is further extended to consider fuzzy partial backlogging factor. Goni and Maheswari (2010) discussed the retailer's ordering policy under two levels of delay payments considering the demand and the selling price as triangular fuzzy numbers. They used graded mean integration representation method for defuzzification.

Lee and Yao (1998) developed an economic production quantity (EPQ) model in which the demand and the production quantity are assumed to be fuzzy. Lo et al. (2007) presented an EPQ model which includes uncertain factors like unreliability of the machineries, flaw of the products or shortage caused by reworked process. They used fuzzy analysis hierarchy procedure (AHP) to calculate the set-up, holding and internal failure costs which affect the optimum production quantity. Halim et al. (2010) addressed the lot sizing problem in an unreliable production system with stochastic machine breakdown and fuzzy repair time. They defuzzified the cost per unit time using the signed distance method. Mahata and Goswami (2006) developed a fuzzy production-inventory model with permissible delay in payment. They assumed the demand and the production rates as fuzzy numbers and defuzzified the associated cost in the fuzzy sense using extension principle. Hsieh (2002) considered two fuzzy production-inventory models: one for crisp production quantity with fuzzy parameters and the other one for fuzzy production quantity. He used the graded mean integration representation method for defuzzifying the fuzzy total cost.

Production of defective items in any manufacturing industry is a natural phenomenon. The number of defectives may have a change from one lot to another that cannot be assessed by a crisp value. If the uncertainty of the product flaw is treated as random then the estimation from the historical data of the value(s) of the parameter(s) involved in the associated probability distribution may not always be accurate. Chen and Chang (2008) developed a fuzzy economic production quantity (EPQ) model with defective productions that cannot be repaired. In this model, they considered a fuzzy opportunity cost and trapezoidal fuzzy costs under crisp production quantity or fuzzy production. Halim et al. (2009) developed an EPQ model in which the fraction of defective items produced after process shift is characterized by a fuzzy number. The production rate and demand rate are being known constants. In another attempt, they assumed that the fraction of defective items follow an exponential probability distribution where the parameter of the distribution is a fuzzy number. Similar to the defective item production rate, it may be difficult to search for an appropriate probability distribution for the annual demand rate and also to estimate the parameter(s) involved in the probability distribution. It is rather easier to locate the annual demand in an interval. So, to capture the real situation better, this paper considers the production and demand rates as fuzzy numbers besides fuzzy defective item production rate. The paper is organized as follows. Notations and assumptions for the proposed models are given in the next Section. The crisp model is presented in Section 3 for better understanding of the production planning problem. Section 4 develops fuzzy model with fuzzy defective item production rate and stochastic demand rate. This fuzzy model is also extended to consider fuzziness in the 
demand rate. Numerical examples are provided in Section 5 to illustrate the developed models and to examine the sensitivity of the model parameters. Finally, in Section 6, some concluding remarks are given.

\section{Notations and Assumptions}

The following notations are used throughout the paper:

$T(>0)$ : scheduling period

$t_{1}(<T)$ : production run time during the scheduling period $T$

$d(>0)$ : annual demand rate

$p(>d)$ : production rate

$X:$ random variable denoting the time to process shift

$f_{X}($.$) : probability density function of X$

$E(N)$ : expected number of defective items produced during a production run

$K(>0)$ : fixed cost per production batch

$h(>0)$ : holding cost per unit item per unit time

$c(>0)$ : defective item cost per unit item.

$\beta$ : a constant fraction, $\quad 0<\beta<1$

$\gamma$ : defective item production rate, $0 \leq \gamma \leq 1$

To develop the proposed models the following assumptions are made:

(1) The production system which is operated by a single unit produces a single item.

(2) The production process is always in in-control state at the beginning of each production run.

(3) The process may shift from the in-control state to the out-of-control state at any random time when some defective items are produced.

(4) The elapsed time before process shift follows an exponential distribution with probability density function

$$
f_{X}(t)=\left\{\begin{array}{l}
\lambda e^{-\lambda t}, \quad 0 \leq t \leq t_{1} ; \lambda>0 \\
0, \quad t>t_{1}
\end{array}\right.
$$

(5) Defective items are neither repaired nor replaced i.e. those are scrapped. 
(6) Shortages are not permitted.

(7) Production rate $(p)$ is dependent on the demand rate $(d)$ and is connected by the relation: $p=\frac{1}{\beta} d, \quad 0<\beta<1$

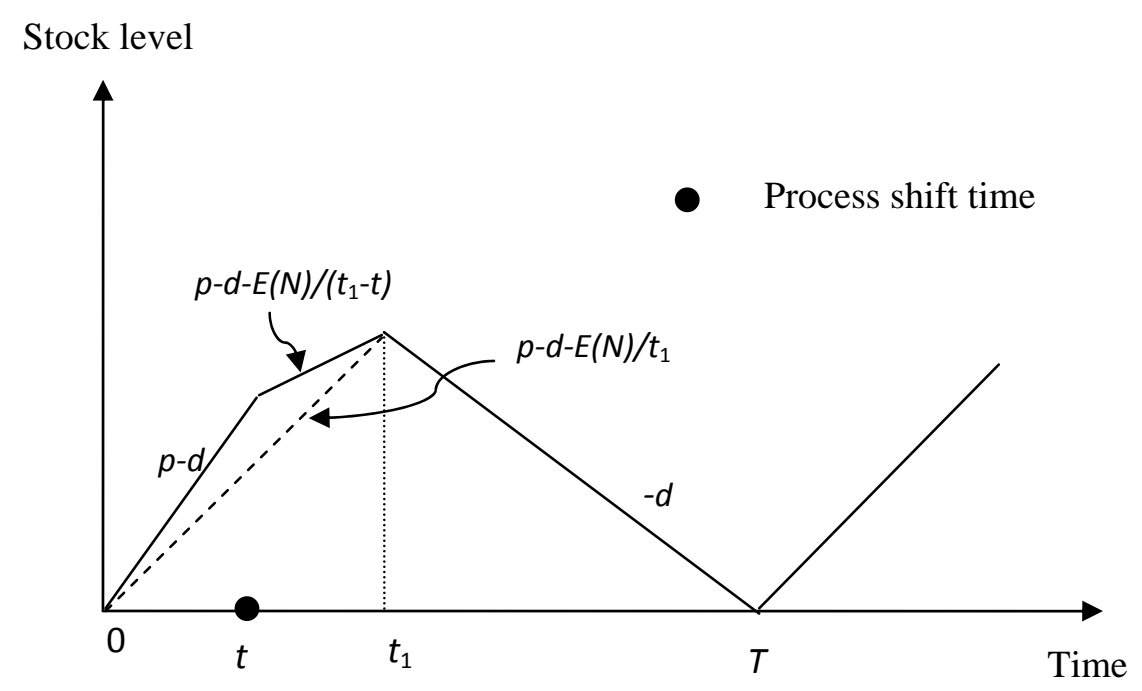

Fig. 1. Schematic diagram of the proposed production-inventory model

\section{Formulation of the Crisp Model}

To derive the inventory cost function for the first scheduling period $T$, we divide the time interval $[0, T]$ into two parts: $\left[0, t_{1}\right]$ and $\left[t_{1}, T\right]$. The production starts at time $t=0$ and stops at time $t=t_{1}$. So, stock builds up during the period $\left[0, t_{1}\right]$ and declines during the period $\left[t_{1}, T\right]$. The inventory path pattern is depicted in Fig 1. If $I_{1}(t)$ and $I_{2}(t)$ denote, respectively, the inventory levels at any time during the time periods $\left[0, t_{1}\right]$ and $\left[t_{1}, T\right]$, then the differential equations representing the inventory status are given by

$\frac{d I_{1}(t)}{d t}=p-d-\frac{E(N)}{t_{1}}, 0 \leq t \leq t_{1}$ with $I_{1}(0)=0$,

$\frac{d I_{2}(t)}{d t}=-d, t_{1} \leq t \leq T$ with $I_{2}(T)=0$,

where $E(N)$, the expected number of defective items produced during the production run is calculated as given below:

If the process shifts at time $t\left(0 \leq t \leq t_{1}\right)$ then the total number of items produced after process shift is $p\left(t_{1}-t\right)$. Hence, the expected number of defective items produced during the production run is

$E(N)=\int_{0}^{\infty} \gamma p\left(t_{1}-t\right) f_{X}(t) d t=\int_{0}^{t_{1}} \gamma p\left(t_{1}-t\right) \lambda e^{-\lambda t} d t=\frac{\not p}{\lambda}\left[e^{-\lambda t_{1}}+\lambda t_{1}-1\right]$. 
Using (4) in (2) and then solving the differential equations (2) and (3), we obtain

$$
\begin{aligned}
& I_{1}(t)=(p-d) t-\frac{\gamma p}{\lambda t_{1}}\left[e^{-\lambda t_{1}}+\lambda t_{1}-1\right] t, \quad 0 \leq t \leq t_{1}, \\
& I_{2}(t)=d(T-t), \quad t_{1} \leq t \leq T .
\end{aligned}
$$

Therefore, inventory holding cost is as follows,

$$
h\left[\int_{0}^{t_{1}} I_{1}(t) d t+\int_{t_{1}}^{T} I_{2}(t) d t\right]=\frac{h}{2 \lambda}\left[\lambda p t_{1}^{2}+\lambda d T^{2}-2 \lambda d t_{1} T-\gamma p t_{1}\left(e^{-\lambda t_{1}}+\lambda t_{1}-1\right)\right],
$$

and defective item cost is $c E(N)=\frac{c \gamma p}{\lambda}\left(e^{-\lambda t_{1}}+\lambda t_{1}-1\right)$.

The total cost per unit time $(W)$ which is the time average of the sum of set up cost, holding cost and defective item cost is given by

$W\left(t_{1}\right)=\frac{K}{T}+\frac{1}{2 \lambda T}\left[h\left\{\lambda p t_{1}^{2}+\lambda d T\left(T-2 t_{1}\right)\right\}+\not p\left(2 c-h t_{1}\right)\left(e^{-\lambda t_{1}}+\lambda t_{1}-1\right)\right]$.

Since $I_{1}\left(t_{1}\right)=I_{2}\left(t_{1}\right)$, therefore, we have $T=\frac{1}{d \lambda}\left\{\lambda p t_{1}-\not p\left(e^{-\lambda t_{1}}+\lambda t_{1}-1\right)\right\}$.

Rearranging the terms, Eq.(7) can be rewritten as

$W\left(t_{1}\right)=\frac{K}{T}+\frac{p}{2 \lambda T}\left[\lambda h t_{1}^{2}+\gamma\left(2 c-h t_{1}\right)\left(e^{-\lambda t_{1}}+\lambda t_{1}-1\right)\right]+\frac{d h}{2}\left(T-2 t_{1}\right)$.

Now substituting $p=\frac{1}{\beta} d$ and $T=\frac{1}{d \lambda}\left\{\lambda p t_{1}-\gamma p\left(e^{-\lambda t_{1}}+\lambda t_{1}-1\right)\right\}$ in the above equation, we get

$$
\begin{aligned}
& W\left(t_{1}\right)=\frac{K \lambda \beta}{\lambda t_{1}-\gamma\left(e^{-\lambda t_{1}}+\lambda t_{1}-1\right)}+\frac{\lambda d p}{2 \lambda p\left\{\lambda t_{1}-\gamma\left(e^{-\lambda t_{1}}+\lambda t_{1}-1\right)\right\}}\left[-\lambda t_{1}\left(2 c-h t_{1}\right)+2 c \lambda t_{1}+\gamma\left(2 c-h t_{1}\right)\left(e^{-\lambda t_{1}}+\lambda t_{1}-1\right)\right] \\
& +\frac{d h}{2 \lambda \beta}\left\{\lambda t_{1}-\gamma\left(e^{-\lambda t_{1}}+\lambda t_{1}-1\right)\right\}-d h t_{1},
\end{aligned}
$$

which after simplification gives

$$
W\left(t_{1}\right)=-\frac{d}{2}\left(2 c+h t_{1}\right)+\frac{\lambda\left(c d t_{1}+K \beta\right)}{\lambda t_{1}-\gamma\left(e^{-\lambda t_{1}}+\lambda t_{1}-1\right)}+\frac{d h}{2 \lambda \beta}\left\{\lambda t_{1}-\gamma\left(e^{-\lambda t_{1}}+\lambda t_{1}-1\right)\right\} .
$$

The objective of this crisp model is to find the optimal production time $t_{1}^{*}$ which minimizes the cost per unit time $W$.

\section{Development of Fuzzy Models}

In this section, we develop two fuzzy models corresponding to the crisp model developed in the previous section. For the fundamental concept of fuzzy sets and numbers, we refer the readers to any standard text book on fuzzy set theory (e.g. Dubois and Prade (1980); Kaufmann and Gupta (1992); Zimmermann (1996); etc.). Furthermore, we introduce the following basic definitions of fuzzy sets 
and numbers (Chen and Hsieh (1999); Hsieh (2002) ) essential for development of the proposed fuzzy models.

Definition 1. Generalized fuzzy number

A generalized fuzzy number $\tilde{A}$ is a fuzzy set on $\mathfrak{R}=(-\infty, \infty)$ whose membership function $\mu_{\tilde{A}}(x)$ satisfies the following conditions:

(i) $\quad \mu_{\tilde{A}}(x)$ is a continuous mapping from $\mathfrak{R}$ to the closed interval $[0,1]$,

(ii) $\mu_{\tilde{A}}(x)=0,-\infty<x \leq a_{1}$,

(iii) $\mu_{\tilde{A}}(x)=L(x)$ is strictly increasing on $\left[a_{1}, a_{2}\right]$,

(iv) $\mu_{\tilde{A}}(x)=w_{A}, \quad a_{2} \leq x \leq a_{3}$,

(v) $\mu_{\tilde{A}}(x)=R(x)$ is strictly decreasing on $\left[a_{3}, a_{4}\right]$,

(vi) $\mu_{\tilde{A}}(x)=0, a_{4} \leq x<\infty$

where $0<w_{A} \leq 1$ and $a_{1}, a_{2}, a_{3}$ and $a_{4}$ are real numbers. The above generalized fuzzy number is denoted by $\tilde{A}=\left(a_{1}, a_{2}, a_{3}, a_{4} ; w_{A}\right)_{L R}$. When $w_{A}=1, \tilde{A}$ becomes $\tilde{A}=\left(a_{1}, a_{2}, a_{3}, a_{4}\right)_{L R}$.

Definition 2. Graded mean integration representation method (Chen and Hsieh, 1999)

The method is based on the integral value of graded mean $h$-level of a generalized fuzzy number for defuzzification. The graded mean $h$-level value of a generalized fuzzy number $\tilde{A}=\left(a_{1}, a_{2}, a_{3}, a_{4} ; w_{A}\right)_{L R}$ is given by $h\left(L^{-1}(h)+R^{-1}(h)\right) / 2$ where $L^{-1}$ and $R^{-1}$ are the inverse functions of $L$ and $R$, respectively. Then, the graded mean integration representation of $\tilde{A}$ with grade $w_{A}$ denoted by $P(\tilde{A})$ is defined as

$$
P(\tilde{A})=\int_{0}^{w_{A}} h\left(\frac{L^{-1}(h)+R^{-1}(h)}{2}\right) d h / \int_{0}^{w_{A}} h d h,
$$

where $0<h \leq w_{A}$ and $0<w_{A} \leq 1$. We use trapezoidal fuzzy numbers for all fuzzy parameters in our proposed fuzzy production inventory models. Let $\widetilde{B}=\left(b_{1}, b_{2}, b_{3}, b_{4}\right)$ be a trapezoidal fuzzy number defined on $\mathfrak{R}=(-\infty, \infty)$. Then, for $\widetilde{B}$, the graded mean integration representation is

$$
P(\tilde{B})=\int_{0}^{1} h\left(\frac{b_{1}+b_{4}+\left(b_{2}-b_{1}-b_{4}+b_{3}\right) h}{2}\right) d h / \int_{0}^{1} h d h=\frac{b_{1}+2 b_{2}+2 b_{3}+b_{4}}{6} .
$$

\section{Definition 3. Fuzzy arithmetic operation under function principle}

Function principle was introduced by Chen (1986). Some fuzzy arithmetical operations of trapezoidal fuzzy numbers under function principle are described as follows:

Let $\tilde{A}=\left(a_{1}, a_{2}, a_{3}, a_{4}\right)$ and $\tilde{B}=\left(b_{1}, b_{2}, b_{3}, b_{4}\right)$ be two trapezoidal fuzzy numbers where $a_{i} \mathrm{~s}$ and $b_{i}$ $\mathrm{s}, i=1,2,3,4$ are real numbers. Then 
(i) $\tilde{A} \oplus \widetilde{B}=\left(a_{1}+b_{1}, a_{2}+b_{2}, a_{3}+b_{3}, a_{4}+b_{4}\right)$.

(ii) $-\widetilde{B}=\left(-b_{4},-b_{3},-b_{2},-b_{1}\right)$.

(iii) $\tilde{A} \Theta \widetilde{B}=\left(a_{1}-b_{4}, a_{2}-b_{3}, a_{3}-b_{2}, a_{4}-b_{1}\right)$.

(iv) $\tilde{A} \otimes \widetilde{B}=\left(c_{1}, c_{2}, c_{3}, c_{4}\right)$

where $E=\left\{a_{1} b_{1}, a_{1} b_{4}, a_{4} b_{1}, a_{4} b_{4}\right\}, E_{1}=\left\{a_{2} b_{2}, a_{2} b_{3}, a_{3} b_{2}, a_{3} b_{3}\right\}, c_{1}=\min E, \quad c_{2}=\min E_{1}, c_{3}=$ $\max E_{1}, c_{4}=\max E$. If $a_{i} \mathrm{~s}$ and $b_{i} \mathrm{~s}, i=1,2,3,4$ are all non zero positive real numbers, then $\tilde{A} \otimes \widetilde{B}=\left(a_{1} b_{1}, a_{2} b_{2}, a_{3} b_{3}, a_{4} b_{4}\right)$.

(v) $1 / \widetilde{B}=\widetilde{B}^{-1}=\left(1 / b_{4}, 1 / b_{3}, 1 / b_{2}, 1 / b_{1}\right)$, where $b_{1}, b_{2}, b_{3}$ and $b_{4}$ are all positive real numbers.

(vi) If $a_{i}$ s and $b_{i} \mathrm{~s}, i=1,2,3,4$ are all non-zero positive real numbers, then the division of $\tilde{A}$ and $\widetilde{B}$, denoted by $\tilde{A} \phi \widetilde{B}$ is defined as $\tilde{A} \phi \widetilde{B}=\left(a_{1} / b_{4}, a_{2} / b_{3}, a_{3} / b_{2}, a_{4} / b_{1}\right)$.

(vii) For $\alpha \in \mathfrak{R}, \alpha \otimes \tilde{A}=\left(\alpha a_{1}, \alpha a_{2}, \alpha a_{3}, \alpha a_{4}\right)$ when $\alpha \geq 0$ and $\alpha \otimes \tilde{A}=\left(\alpha a_{4}, \alpha a_{3}, \alpha a_{2}, \alpha a_{1}\right)$ when $\alpha<0$ where $\phi, \otimes, \Theta$ and $\oplus$ are the fuzzy arithmetical operations under function principle.

\subsection{Model-I with stochastic demand and fuzzy defective rate}

In this sub-section, we develop a fuzzy model with stochastic demand treating the production rate $p$ and the defective item production rate $\gamma$ as fuzzy numbers. Let the annual demand be represented by a random variable $X$ which follows a uniform probability distribution with mean $d$ and a range $d(1-a)$ to $d(1+a), 0 \leq a \leq 1$.

i.e., $\quad f_{X}(x)= \begin{cases}\frac{1}{2 a d}, & d(1-a) \leq x \leq d(1+a), \\ 0, & \text { otherwise. }\end{cases}$

In this case, Eq. (8) takes the form

$W\left(t_{1}\right)=\frac{K \lambda \beta}{\lambda t_{1}-\gamma\left(e^{-\lambda t_{1}}+\lambda t_{1}-1\right)}+\left[\frac{\lambda c t_{1}}{\lambda t_{1}-\gamma\left(e^{-\lambda t_{1}}+\lambda t_{1}-1\right)}+\frac{h}{2 \lambda \beta}\left\{\lambda t_{1}-\gamma\left(e^{-\lambda t_{1}}+\lambda t_{1}-1\right)\right\}-\frac{1}{2}\left(2 c+h t_{1}\right)\right] x$.

Therefore, the expected inventory cost per unit time $W_{0}\left(t_{1}\right)$ is given by

$$
\begin{aligned}
W_{0}\left(t_{1}\right) & =\int_{d(1-a)}^{d(1+a)} W\left(t_{1}\right) f_{X}(x) d x \\
& =\frac{K \lambda \beta}{\lambda t_{1}-\gamma\left(e^{-\lambda t_{1}}+\lambda t_{1}-1\right)}+d\left[\frac{\lambda c t_{1}}{\lambda t_{1}-\gamma\left(e^{-\lambda t_{1}}+\lambda t_{1}-1\right)}+\frac{h}{2 \lambda \beta}\left\{\lambda t_{1}-\gamma\left(e^{-\lambda t_{1}}+\lambda t_{1}-1\right)\right\}-\frac{1}{2}\left(2 c+h t_{1}\right)\right] \\
& =-\frac{d}{2}\left(2 c+h t_{1}\right)+\frac{\lambda\left(c d t_{1}+K \beta\right)}{\lambda t_{1}-\gamma\left(e^{-\lambda t_{1}}+\lambda t_{1}-1\right)}+\frac{d h}{2 \lambda \beta}\left\{\lambda t_{1}-\gamma\left(e^{-\lambda t_{1}}+\lambda t_{1}-1\right)\right\}
\end{aligned}
$$


Suppose that $\beta$ and $\gamma$ are two generalized fuzzy numbers, say $\tilde{\beta}$ and $\tilde{\gamma}$, respectively. Then, using fuzzy arithmetical operations $\phi, \Theta, \otimes$ and $\oplus$ under function principle, we may rewrite the above equation as

$$
\begin{aligned}
\tilde{W}_{1} \equiv \tilde{W}_{1}\left(t_{1}\right)=- & {\left[(d / 2)\left(2 c+h t_{1}\right)\right] \oplus\left[\left\{\lambda \otimes\left(c d t_{1} \oplus K \otimes \tilde{\beta}\right)\right\}\right.} \\
& \left.\phi\left\{\lambda t_{1} \Theta \tilde{\gamma} \otimes\left(e^{-\lambda t_{1}}+\lambda t_{1}-1\right)\right\}\right] \oplus\left[\{d h \phi(2 \lambda \otimes \tilde{\beta})\} \otimes\left\{\lambda t_{1} \Theta \tilde{\gamma} \otimes\left(e^{-\lambda t_{1}}+\lambda t_{1}-1\right)\right\}\right] .
\end{aligned}
$$

Let us assume $\beta$ and $\gamma$ as two non-negative trapezoidal fuzzy numbers, i.e., $\tilde{\beta}=\left(\beta_{1}, \beta_{2}, \beta_{3}, \beta_{4}\right)$ and $\tilde{\gamma}=\left(\gamma_{1}, \gamma_{2}, \gamma_{3}, \gamma_{4}\right)$, where $\beta_{i}$ s and $\gamma_{i} \mathrm{~s}, i=1,2,3,4$ are determined by the decision maker. Then, the expected cost per unit time is a fuzzy value and we obtain it by formula (11) as

$$
\begin{aligned}
\tilde{W}_{1}=[ & -\frac{d}{2}\left(2 c+h t_{1}\right)+\frac{\lambda\left(c d t_{1}+K \beta_{1}\right)}{\lambda t_{1}-\gamma_{1}\left(e^{-\lambda t_{1}}+\lambda t_{1}-1\right)}+\frac{d h}{2 \lambda \beta_{4}}\left\{\lambda t_{1}-\gamma_{4}\left(e^{-\lambda t_{1}}+\lambda t_{1}-1\right)\right\}, \\
& -\frac{d}{2}\left(2 c+h t_{1}\right)+\frac{\lambda\left(c d t_{1}+K \beta_{2}\right)}{\lambda t_{1}-\gamma_{2}\left(e^{-\lambda t_{1}}+\lambda t_{1}-1\right)}+\frac{d h}{2 \lambda \beta_{3}}\left\{\lambda t_{1}-\gamma_{3}\left(e^{-\lambda t_{1}}+\lambda t_{1}-1\right)\right\}, \\
& -\frac{d}{2}\left(2 c+h t_{1}\right)+\frac{\lambda\left(c d t_{1}+K \beta_{3}\right)}{\lambda t_{1}-\gamma_{3}\left(e^{-\lambda t_{1}}+\lambda t_{1}-1\right)}+\frac{d h}{2 \lambda \beta_{2}}\left\{\lambda t_{1}-\gamma_{2}\left(e^{-\lambda t_{1}}+\lambda t_{1}-1\right)\right\}, \\
& \left.-\frac{d}{2}\left(2 c+h t_{1}\right)+\frac{\lambda\left(c d t_{1}+K \beta_{4}\right)}{\lambda t_{1}-\gamma_{4}\left(e^{-\lambda t_{1}}+\lambda t_{1}-1\right)}+\frac{d h}{2 \lambda \beta_{1}}\left\{\lambda t_{1}-\gamma_{1}\left(e^{-\lambda t_{1}}+\lambda t_{1}-1\right)\right\}\right] .
\end{aligned}
$$

We defuzzify $\tilde{W}_{1}$ using the graded mean integration representation method (see Chen and Hsieh (1999); Hsieh (2002)) and estimate cost per unit time in the fuzzy sense by formula (9) as

$$
\begin{aligned}
P\left(\tilde{W}_{1}\right)=-\frac{d}{2}\left(2 c+h t_{1}\right)+\frac{\lambda}{6}\left[\frac{c d t_{1}+K \beta_{1}}{\lambda t_{1}-\gamma_{1}\left(e^{-\lambda t_{1}}+\lambda t_{1}-1\right)}+\frac{2\left(c d t_{1}+K \beta_{2}\right)}{\lambda t_{1}-\gamma_{2}\left(e^{-\lambda t_{1}}+\lambda t_{1}-1\right)}\right. \\
\left.+\frac{2\left(c d t_{1}+K \beta_{3}\right)}{\lambda t_{1}-\gamma_{3}\left(e^{-\lambda t_{1}}+\lambda t_{1}-1\right)}+\frac{c d t_{1}+K \beta_{4}}{\lambda t_{1}-\gamma_{4}\left(e^{-\lambda t_{1}}+\lambda t_{1}-1\right)}\right] \\
+\frac{d h}{12 \lambda}\left[\frac{1}{\beta_{4}}\left\{\lambda t_{1}-\gamma_{4}\left(e^{-\lambda t_{1}}+\lambda t_{1}-1\right)\right\}+\frac{2}{\beta_{3}}\left\{\lambda t_{1}-\gamma_{3}\left(e^{-\lambda t_{1}}+\lambda t_{1}-1\right)\right\}\right. \\
\left.+\frac{2}{\beta_{2}}\left\{\lambda t_{1}-\gamma_{2}\left(e^{-\lambda t_{1}}+\lambda t_{1}-1\right)\right\}+\frac{1}{\beta_{1}}\left\{\lambda t_{1}-\gamma_{1}\left(e^{-\lambda t_{1}}+\lambda t_{1}-1\right)\right\}\right] .
\end{aligned}
$$

If the objective function (13) is convex, then any suitable one dimensional search technique can be applied to find numerically the optimal value $t_{1}{ }^{*}$ which minimizes $P\left(\tilde{W}_{1}\right)$.

Proposition: There exists at least one local optimal value of $P\left(\tilde{W}_{1}\right)$ if $\beta+\gamma<1$.

Proof: Differentiating equation (13) with respect to $t_{1}$, we obtain the optimality condition for minimization of $P\left(\tilde{W}_{1}\right)$ as:

$$
g\left(t_{1}\right) \equiv \frac{d P}{d t_{1}}=-\frac{d h}{2}+\frac{\lambda}{6}\left[\sum_{i=1}^{4} \frac{c d A_{i}-\left(c d t_{1}+K \beta_{i}\right) \frac{d A_{i}}{d t_{1}}}{A_{i}^{2}}+\sum_{i=2}^{3} \frac{c d A_{i}-\left(c d t_{1}+K \beta_{i}\right) \frac{d A_{i}}{d t_{1}}}{A_{i}^{2}}\right]
$$




$$
+\frac{d h}{12 \lambda}\left[\sum_{i=1}^{4} \frac{1}{\beta_{i}} \frac{d A_{i}}{d t_{1}}+\sum_{i=2}^{3} \frac{1}{\beta_{i}} \frac{d A_{i}}{d t_{1}}\right]=0
$$

where $A_{i}=\lambda t_{1}\left(1-\gamma_{i}\right)+\gamma_{i}\left(1-e^{-\lambda t_{1}}\right), i=1,2,3,4$.

Clearly, $g(0)=-\infty$

and

$g(\infty)=\frac{d h}{12}\left[\frac{1-\gamma_{1}}{\beta_{1}}+\frac{2\left(1-\gamma_{2}\right)}{\beta_{2}}+\frac{2\left(1-\gamma_{3}\right)}{\beta_{3}}+\frac{1-\gamma_{4}}{\beta_{4}}-6\right]-\lim _{t_{1} \rightarrow \infty}\left[\sum_{i=1}^{4} \frac{\left(c d t_{1}+K \beta_{i}\right) \frac{d A_{i}}{d t_{1}}}{A_{i}^{2}}+\sum_{i=2}^{3} \frac{\left(c d t_{1}+K \beta_{i}\right) \frac{d A_{i}}{d t_{1}}}{A_{i}^{2}}\right]$.

Now,

$$
\begin{aligned}
& \lim _{t_{1} \rightarrow \infty} \frac{\left(c d t_{1}+K \beta_{1}\right) \frac{d A_{1}}{d t_{1}}}{A_{1}^{2}}\left(\frac{\infty}{\infty} \text { form }\right) \\
= & \lim _{t_{1} \rightarrow \infty} \frac{c d \frac{d A_{1}}{d t_{1}}-\lambda^{2} \gamma_{1} e^{-\lambda t_{1}}\left(c d t_{1}+K \beta_{1}\right)}{2 A_{1} \frac{d A_{1}}{d t_{1}}} \text { (using L' Hospital's rule) } \\
= & \lim _{t_{1} \rightarrow \infty} \frac{-2 c d \lambda^{2} \gamma_{1} e^{-\lambda t_{1}}+\lambda^{3} \gamma_{1} e^{-\lambda t_{1}}\left(c d t_{1}+K \beta_{1}\right)}{2\left(\frac{d A_{1}}{d t_{1}}\right)^{2}-2 \lambda^{2} \gamma_{1} e^{-\lambda t_{1}} A_{1}} \text { (using L' Hospital's rule) } \\
= & \frac{0}{\infty}=0 .
\end{aligned}
$$

Similarly, it can be shown that

$$
\lim _{t_{1} \rightarrow \infty} \frac{\left(c d t_{1}+K \beta_{i}\right) \frac{d A_{i}}{d t_{1}}}{A_{i}^{2}}=0 \text { for } i=2,3,4 .
$$

Therefore, $g(\infty)=\frac{d h}{12}\left[\frac{1-\gamma_{1}}{\beta_{1}}+\frac{2\left(1-\gamma_{2}\right)}{\beta_{2}}+\frac{2\left(1-\gamma_{3}\right)}{\beta_{3}}+\frac{1-\gamma_{4}}{\beta_{4}}-6\right]>0$ if $\frac{1-\gamma_{4}}{\beta_{4}}>1$,

because $\beta_{1}, \beta_{2}, \beta_{3}<\beta_{4}$ and $\gamma_{1}, \gamma_{2}, \gamma_{3}<\gamma_{4}$ implying that $\frac{1-\gamma_{i}}{\beta_{i}}>1$ for $i=1$, 2, 3 when $\beta_{4}+\gamma_{4}<1$. Hence the proposition is proved.

\subsection{Model-II with fuzzy demand and fuzzy defective rate}

In this sub-section, we will extend the previous model by assuming the demand rate $d$ as fuzzy. The reason behind this assumption is that it is sometimes easier to locate annual demand rate in an interval rather than finding an appropriate probability distribution for it. We fuzzify $d$ by assuming it to be a non-negative trapezoidal fuzzy member $\tilde{d}=\left(d_{1}, d_{2}, d_{3}, d_{4}\right)$ where $d_{1}, d_{2}, d_{3}$ and $d_{4}$ are determined by the decision maker. In this case, the expected fuzzy cost $\tilde{W}_{2} \equiv \tilde{W}_{2}\left(t_{1}\right)$ per unit time can be obtained by formula (11) as: 


$$
\begin{aligned}
\tilde{W}_{2}= & -\frac{d_{4}}{2}\left(2 c+h t_{1}\right)+\frac{\lambda\left(c d_{1} t_{1}+K \beta_{1}\right)}{\lambda t_{1}-\gamma_{1}\left(e^{-\lambda t_{1}}+\lambda t_{1}-1\right)}+\frac{d_{1} h}{2 \lambda \beta_{4}}\left\{\lambda t_{1}-\gamma_{4}\left(e^{-\lambda t_{1}}+\lambda t_{1}-1\right)\right\}, \\
& -\frac{d_{3}}{2}\left(2 c+h t_{1}\right)+\frac{\lambda\left(c d_{2} t_{1}+K \beta_{2}\right)}{\lambda t_{1}-\gamma_{2}\left(e^{-\lambda t_{1}}+\lambda t_{1}-1\right)}+\frac{d_{2} h}{2 \lambda \beta_{3}}\left\{\lambda t_{1}-\gamma_{3}\left(e^{-\lambda t_{1}}+\lambda t_{1}-1\right)\right\}, \\
& -\frac{d_{2}}{2}\left(2 c+h t_{1}\right)+\frac{\lambda\left(c d_{3} t_{1}+K \beta_{3}\right)}{\lambda t_{1}-\gamma_{3}\left(e^{-\lambda t_{1}}+\lambda t_{1}-1\right)}+\frac{d_{3} h}{2 \lambda \beta_{2}}\left\{\lambda t_{1}-\gamma_{2}\left(e^{-\lambda t_{1}}+\lambda t_{1}-1\right)\right\}, \\
& \left.-\frac{d_{1}}{2}\left(2 c+h t_{1}\right)+\frac{\lambda\left(c d_{4} t_{1}+K \beta_{4}\right)}{\lambda t_{1}-\gamma_{4}\left(e^{-\lambda t_{1}}+\lambda t_{1}-1\right)}+\frac{d_{4} h}{2 \lambda \beta_{1}}\left\{\lambda t_{1}-\gamma_{1}\left(e^{-\lambda t_{1}}+\lambda t_{1}-1\right)\right\}\right] .
\end{aligned}
$$

Similar to the previous sub-section, we defuzzify $\tilde{W}_{2}$ using the graded mean integration representation method by formula (9) as

$$
\begin{aligned}
P\left(\tilde{W}_{2}\right)= & -\frac{1}{12}\left(2 c+h t_{1}\right)\left(d_{1}+2 d_{2}+2 d_{3}+d_{4}\right) \\
& +\frac{\lambda}{6}\left[\frac{c d_{1} t_{1}+K \beta_{1}}{\lambda t_{1}-\gamma_{1}\left(e^{-\lambda t_{1}}+\lambda t_{1}-1\right)}+\frac{2\left(c d_{2} t_{1}+K \beta_{2}\right)}{\lambda t_{1}-\gamma_{2}\left(e^{-\lambda t_{1}}+\lambda t_{1}-1\right)}\right. \\
& \left.+\frac{2\left(c d_{3} t_{1}+K \beta_{3}\right)}{\lambda t_{1}-\gamma_{3}\left(e^{-\lambda t_{1}}+\lambda t_{1}-1\right)}+\frac{c d_{4} t_{1}+K \beta_{4}}{\lambda t_{1}-\gamma_{4}\left(e^{-\lambda t_{1}}+\lambda t_{1}-1\right)}\right] \\
& +\frac{h}{12 \lambda}\left[\frac{d_{1}}{\beta_{4}}\left\{\lambda t_{1}-\gamma_{4}\left(e^{-\lambda t_{1}}+\lambda t_{1}-1\right)\right\}+\frac{2 d_{2}}{\beta_{3}}\left\{\lambda t_{1}-\gamma_{3}\left(e^{-\lambda t_{1}}+\lambda t_{1}-1\right)\right\}\right. \\
& \left.+\frac{2 d_{3}}{\beta_{2}}\left\{\lambda t_{1}-\gamma_{2}\left(e^{-\lambda t_{1}}+\lambda t_{1}-1\right)\right\}+\frac{d_{4}}{\beta_{1}}\left\{\lambda t_{1}-\gamma_{1}\left(e^{-\lambda t_{1}}+\lambda t_{1}-1\right)\right\}\right] .
\end{aligned}
$$

The objective here is to find the optimal value of $t_{1}$ which minimizes $\tilde{W}_{2}\left(t_{1}\right)$. Similar to Model-I, it is difficult to prove the convexity property of the cost function $P\left(\tilde{W}_{2}\right)$ analytically. However, an appropriate search technique can be applied to find the optimal solution numerically.

\section{Numerical Results}

In order to illustrate the numerical outcomes of the models developed in Sections 3 and 4, we consider the following input data: $K=600, d=80, h=1, c=5, \lambda=0.5, \beta=0.5, \gamma=0.15$ in appropriate units. Using the numerical computational software Mathematica, we obtain the optimal crisp value of the production time $t_{1}{ }^{*}$ as 2.84289 units and the corresponding expected cost per unit time $W\left(t_{1 c}{ }^{*}\right)$ as 241.352 units. For the fuzzy models, instead of taking $\beta=0.5$ we now take $\beta$ around 0.5. Also, we consider the defective rate around $\gamma=0.15$ i.e. good-quality rate is about 0.85 . The optimal production time and the minimum expected cost per unit time in the fuzzy sense corresponding to the fuzzy Models I \& II are presented in Table 1 . Here, we use a general rule to transfer the linguistic data, "greater or less than $Z$ " or "around $Z$ ”, into trapezoidal fuzzy number as: 
"greater or less than $Z$ " or "around $Z$ " $=(0.9 Z, 0.95 Z, 1.05 Z, 1.1 Z)$. Then, by the above rule, the fuzzy parameters in this example can be transferred as follows:

Fuzzy demand rate=“greater or less than $80 ”=\tilde{d}=\left(d_{1}, d_{2}, d_{3}, d_{4}\right)=(72,76,84,88)$,

Fuzzy $\beta=$ “around $0.5 ”=\widetilde{\beta}=\left(\beta_{1}, \beta_{2}, \beta_{3}, \beta_{4}\right)=(0.450,0.475,0.525,0.550)$,

Fuzzy $\gamma=$ "around $0.15 "=\tilde{\gamma}=\left(\gamma_{1}, \gamma_{2}, \gamma_{3}, \gamma_{4}\right)=(0.1350,0.1425,0.1575,0.1650)$

Table 1

Optimal results of the proposed fuzzy models

\begin{tabular}{lcc}
\hline Model & $t_{1}^{*}$ & $W_{1}{ }^{*}\left(t_{1}^{*}\right)$ \\
\hline I & 2.8087 & 242.958 \\
II & 2.8067 & 243.848 \\
\hline
\end{tabular}

\subsection{Sensitivity Analysis}

We will now perform the sensitivity analysis to examine the effects of changes in the input parameters $K, d, h, c, \lambda, \beta$ and $\gamma$ on the optimal results obtained in Model-I. At first, we find the optimal values of $t_{1}$ and $W_{1}$ by changing each of the parameters by $50 \%, 20 \%,-20 \%$ and $-50 \%$, taking one parameter at a time and keeping the remaining parameters unchanged. Then we calculate the percentage change of $t_{1}^{*}$ and $W_{1}^{*}$ with the base value. The results are shown in Table 2. The following observations can be made from the sensitivity analysis:

(i) From Table 2, we see that the percentage change in the cost is almost equal for small changes (both positive and negative) of all the parameters.

(ii) The model is moderately sensitive to the changes in the parameters $K, d, h$ and $\beta$.

(iii) The model shows low sensitivity with respect to the parameters $c, \lambda$ and $\gamma$.

(iv) $t_{1}^{*}$ is highly sensitive with respect to the parameter $\beta$.

It is also noted that $W_{1}^{*}\left(t_{1}^{*}\right)$ increases with the increase in all the parameters $K, d, h, c, \lambda$ and $\gamma$ whereas it decreases with the increase in the parameter $\beta$. The reason is that when $\beta$ increases, the production rate decreases (by Eq. (1)). As a result, the machine produces less in quantity. Consequently, the defective items produced are also less. Thus, the lower holding cost and defective item cost result in a decrease in the average cost. Similar characteristics are observed in Model-II. 


\section{Table 2}

Sensitivity analysis with respect to the parameters in Model-I

\begin{tabular}{|c|c|c|c|}
\hline Parameter & $\%$ change in parameter & $\%$ change in $t_{1}^{*}$ & $\%$ change in $W_{1}\left(t_{1}^{*}\right)$ \\
\hline & +50 & 26.6686 & 20.9332 \\
\hline \multirow[t]{4}{*}{ K } & +20 & 11.1902 & 8.9472 \\
\hline & -20 & - 12.1084 & -10.0026 \\
\hline & -50 & -32.7970 & - 28.0464 \\
\hline & +50 & -20.8459 & 23.8070 \\
\hline \multirow[t]{4}{*}{$d$} & +20 & -10.0150 & 10.1038 \\
\hline & -20 & 13.8690 & - 11.1583 \\
\hline & -50 & 49.8861 & -30.8769 \\
\hline & +50 & -17.6284 & 18.5250 \\
\hline \multirow[t]{4}{*}{$h$} & +20 & - 8.2999 & 7.8046 \\
\hline & -20 & 11.0489 & - 8.5031 \\
\hline & -50 & 37.8951 & - 23.1707 \\
\hline & +50 & -5.1280 & 6.0327 \\
\hline \multirow[t]{4}{*}{$C$} & +20 & -2.1120 & 2.4395 \\
\hline & -20 & 2.1960 & -2.4753 \\
\hline & -50 & 5.6510 & -6.2571 \\
\hline & +50 & 2.8825 & 2.7342 \\
\hline \multirow[t]{4}{*}{$\lambda$} & +20 & 1.1080 & 1.2117 \\
\hline & -20 & - 1.0055 & - 1.3928 \\
\hline & -50 & -2.2512 & -3.8682 \\
\hline & +50 & 177.4230 & - 27.9472 \\
\hline \multirow[t]{3}{*}{$\beta$} & +20 & 41.3540 & - 9.0481 \\
\hline & -20 & - 28.1796 & 7.1708 \\
\hline & -50 & -60.3870 & 16.2361 \\
\hline \multirow[t]{4}{*}{$\gamma$} & +50 & 0.7747 & 5.4754 \\
\hline & +20 & 0.4262 & 2.1106 \\
\hline & -20 & -0.5469 & - 2.0119 \\
\hline & -50 & - 1.5406 & -4.8576 \\
\hline
\end{tabular}




\section{Concluding Remarks}

Uncertainties in demand, production, defective item production etc. are inherent in any unreliable manufacturing system. In this article, we have developed two production planning models for an unreliable manufacturing system. In the first model, the demand rate was assumed to be stochastic whereas in the second model, the demand rate was assumed to be fuzzy. The production rate was proportional to the demand rate where the constant of proportionality was assumed to be a fuzzy number. Well known trapezoidal membership function was used for all the fuzzy numbers. Though several approaches viz. random number technique, probability theory including fuzzy set theory have the capability to capture uncertainties arising in inventory system but it is still difficult to identify which technique performs better. However, the advantage of the fuzzy approach is that it relaxes the rigid assumptions such as constant defective rate, constant demand rate etc. Also, it eases the difficulties in searching for suitable probability distribution function to represent the random behavior of uncontrollable variables.

\section{Acknowledgment}

The authors are thankful to anonymous referees for their helpful comments and suggestions on the earlier versions of the manuscript. The second author gratefully acknowledges the Research Grant (2009-2011) support provided by Jadavpur University.

\section{References}

Bellman, R. E. \& Zadeh, L. A. (1970). Decision-making in a fuzzy environment. Management Science, 17, B141-B164.

Chen, S. H. (1986). Operations on fuzzy numbers with function principle. Tamkang Journal of Management Science, 6 (1), 13-25.

Chen, S. H. \& Chang, S. M. (2008). Optimization of fuzzy production inventory model with unrepairable defective products. International Journal of Production Economics, 113, 887-894.

Chen, S. H. \& Hsieh, C. H (1999). Graded mean integration representation of generalized fuzzy number. Journal of Chinese Fuzzy Systems, 5 (2), 1-7.

Dubois, D. \& Prade, H. (1980), Fuzzy Sets and Systems: Theory and Applications, Academic Press, New York.

Gen, M., Tsujimura, Y. \& Zheng, P.Z. (1997). An application of fuzzy set theory to inventory control models. Computers and Industrial Engineering, 33, 553-556.

Goni, A. \& Maheswari, S. (2010). Supply chain model for the retailer's ordering policy under two levels of delay payments in fuzzy environment. Applied Mathematical Sciences, 4, 1155-1164.

Halim, K.A., Giri, B.C. \& Chaudhuri, K.S. (2008). Fuzzy economic order quantity model for perishable items with stochastic demand, partial backlogging and fuzzy deteriorating rate. International Journal of Operational Research, 3, 77-96.

Halim, K.A., Giri, B.C. \& Chaudhuri, K.S. (2008). Fuzzy EPQ models for an imperfect production system. International Journal of Systems Science, 40(1), 45-52. 
Halim, K.A., Giri, B.C. \& Chaudhuri, K.S.(2010). Lot sizing in an unreliable manufacturing system with fuzzy demand and repair time. International Journal of Industrial and Systems Engineering, 5, 485-500.

Harris, F. (1915). Operations and Cost, Factory Management Service, Chicago: A.W. Shaw Co.

Hsieh, C.H. (2002). Optimization of fuzzy production inventory models. Information Sciences, 146, 29-40.

Ishii, H. \& Konno, T. (1998). A stochastic inventory problem with fuzzy shortage cost. European Journal of Operational Research, 106, 90-94.

Kaufmann, A. \& Gupta, M. M. (1992), Introduction to Fuzzy Arithmetic Theory and Applications. Van Nostrand Reinhold, New York.

Lee, H. M. \& Yao, J. S. (1998). Economic production quantity for fuzzy demand quantity and fuzzy production quantity. European Journal of Operational Research, 109, 203-211.

Lo, C. Y., Leu, J. H. \& Hou, C. I. (2007). A study of the EPQ model using Fuzzy AHP when flaw of the products or unreliable machineries exists, In proceeding of 2007 IEEE International Conference on Industrial Engineering and Engineering Management , pp. 1163-1170.

Mahapatra, N. K. \& Maiti, M. (2006). A fuzzy stochastic approach to multi-objective inventory model of deteriorating items with various types of demand and time dependent holding cost, Journal of the Operational Research Society of India, 43 (2), 117-131.

Mahata, G. C. \& Goswami, A. (2006). Production lot size model with fuzzy production rate and fuzzy demand rate for deteriorating item under permissible delay in payments. Journal of the Operational Research Society of India, 43 (4), 358-375.

Maiti, M. K. \& Maiti, M. (2006). Fuzzy inventory model with two warehouses under possibility constraints, Fuzzy Sets and Systems, 157, 52-73.

Mondal, S. \& Maiti, M. (2002). Multi-item fuzzy EOQ models using genetic algorithm, Computers \& Industrial Engineering, 44, 105-117.

Petrovic, D. \& Sweeney, E. (1994). Fuzzy knowledge-based approach to treating uncertainty in inventory control, Computer Integrated Manufacturing System, 7 (3), 147-152.

Yao, J. S. \& Chiang, J. (2003). Inventory without backorder with fuzzy total cost and fuzzy storing cost defuzzified by centroid and signed distance. European Journal of Operational Research, 148, 401-409.

Zadeh, L. A. (1965). Fuzzy Sets, Information and Control, 8, 338-353.

Zimmermann, H. J. (1996), Fuzzy Set Theory and Its Applications: Third ed., Kluwer Academic Publishers, Dordrecht. 